Conference Proceedings Paper - Entropy

\title{
Neural Wave Interference in Inhibition-Stabilized Networks
}

\author{
Sergey Savel'ev ${ }^{1, *}$ and Sergei Gepshtein ${ }^{2}$ \\ ${ }^{1}$ Department of Physics, Loughborough University, Leicestershire, LE11 3TU, United Kingdom \\ ${ }^{2}$ Center for Neurobiology of Vision, Salk Institute for Biological Studies, 10010 North Torrey Pines \\ Road, La Jolla, CA 92037, USA
}

* Author to whom correspondence should be addressed; S.Saveliev@lboro.ac.uk, +44 (0)1509 223301.

Received: 12 September 2014 / Accepted: 22 October 2014 / Published: 3 November

\begin{abstract}
We study how excitation propagates in chains of inhibition-stabilized neural networks with nearest-neighbor coupling. The excitation generated by local stimuli in such networks propagates across space and time, forming spatiotemporal waves that affect the dynamics of excitation generated by stimuli separated in space and time. These interactions form characteristic interference patterns, manifested as selective preference of the network: for spatial and temporal frequencies of stimulus intensity, for stimulus velocities, and as contextual ("lateral") interactions between stimuli. Such preferences have been previously attributed to distinct specialized mechanisms.
\end{abstract}

Keywords: Vision, neural networks, stimuli interference

PACS classifications: $84.35 .+\mathrm{i}, 42.66 . \mathrm{Si}$

\section{Introduction}

Waves propagating through a medium from difference sources may form patterns. When the wave equations are linear, the waves interfere constructively or destructively, yielding local nodes and antinodes that may retain their spatial positions (as in standing waves) or evolve in space and time (producing dynamic patterns). Such effects were originally studied for acoustic and light waves [1-4] followed by observations of interference for quantum particles [5,6].

More recently, propagation of activity in the neural tissue was studied in terms of the waves of neural excitation $[7,8]$. One may expect that a wave generated in a sensory network at one stimulus location would interfere with the waves generated at other locations. Such "lateral" interactions between stimuli 
are commonly observed in psychophysical and physiological studies of biological sensory systems, where spatially separated stimuli produce patterns of facilitation and suppression in the intervening space [9-11]. But these interactions have not been attributed to neural wave interference. Instead, they have been commonly described in terms of filter characteristics of sensory systems and in terms of long-range neuronal mechanisms [12].

On the description of sensory mechanisms as filters, the response of a biological sensory system to a stimulus is predicted by convolving the stimulus with a kernel whose parameters are estimated in psychophysical [13,14] or physiological [15] studies, or are selected for computational reasons in view of the goals of the visual system (e.g., [16]). Mathematically, this approach is equivalent to describing the system by a set of spatiotemporal differential equations, for which the solutions are found by convolving the simulated inputs with the Green's function of the neural network $[17,18]$.

Here we propose that the commonly observed phenomena of suppression and facilitation of spatially and temporally separated stimuli in biological sensory systems can be understood in terms of interference of neural waves propagating through the network, and described by the spatiotemporal differential equations supporting damped-wave solutions. We investigate the interference patterns created by the waves of excitation in one-dimensional neural chains. We find that such patterns resemble several salient properties of the sensory phenomena that have been attributed to distinct specialized mechanisms. We show that these phenomena can be controlled by a small number of parameters in the interest of making the sensory system selectively tuned to useful properties of the optical environment.

\section{Inhibition-stabilized networks with nearest-neighbor coupling}

Figure 1. Generic circuit. An inhibitory-excitatory neuronal chain with nearest-neighbor coupling [19,20]. The arrows indicate excitatory (red) and inhibitory (blue) interactions between neurons, while currents $i_{E}(l)$ and $i_{I}(l)$ correspond to the inputs into each node generated by the stimulus.

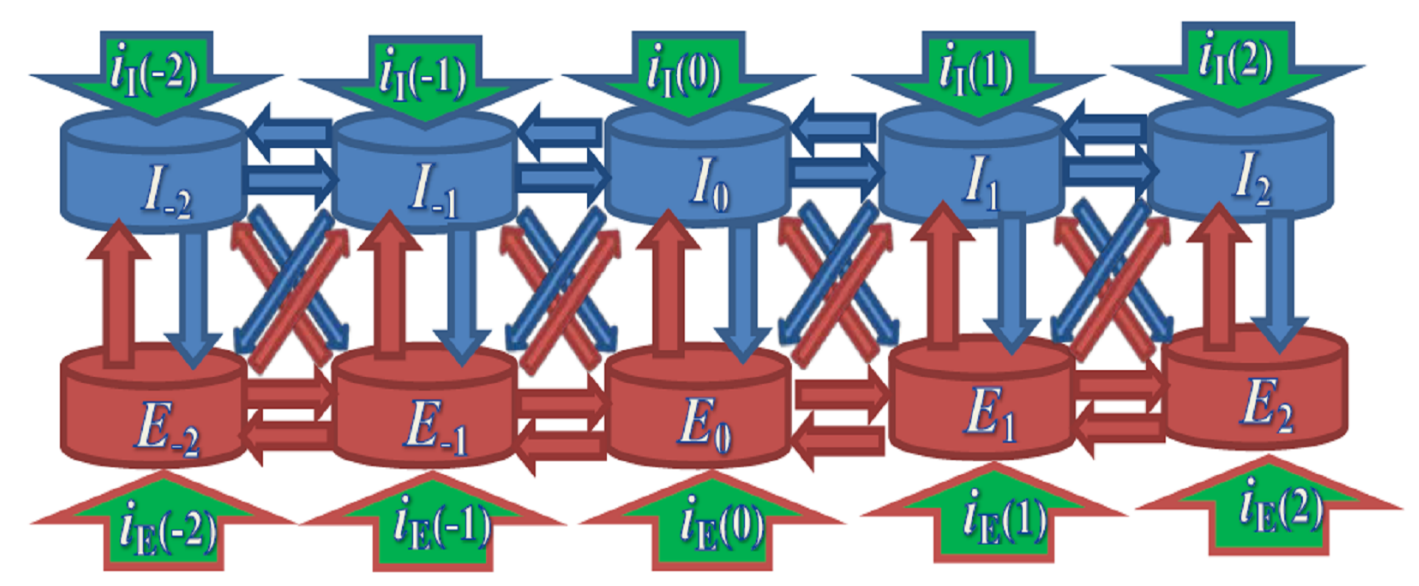


Neural networks underlying sensory processes have been modeled at different levels of abstraction, including the models for local circuitry and for interactions between the local circuits [8,19]. However, phenomena of interference tend to depend more significantly on network geometry and topology (i.e., whether the network is one- or two-dimensional, fractal, etc) than on the finer details of local circuitry: the "node" of the network. To illustrate basic principles of neural-wave interference, here we consider a simple model of a chain of inhibition-excitation nodes with nearest-neighbor interactions (Fig. 1) [20]. We use the Wilson-Cowan model because even a single-node version of it (which consists of one excitatory cell and one inhibitory cell) has proven useful for understanding behavior of small cortical circuits [21], and its rich dynamical properties [22] have been helpful in studies of large-scale neural phenomena $[23,24]$.

Consider a nearest-neighbor chain of Wilson-Cowan nodes [20]:

$$
\begin{aligned}
\tau_{E} \frac{d r_{E}(l)}{d t} & =-r_{E}(l)+g\left(\mathcal{W}_{E}\right) \\
\frac{d r_{I}(l)}{d t} & =-r_{I}(l)+g\left(\mathcal{W}_{I}\right),
\end{aligned}
$$

where

$$
\begin{gathered}
\mathcal{W}_{E}=w_{E E} r_{E}(l)+\tilde{w}_{E E} r_{E}(l+1)+\tilde{w}_{E E} r_{E}(l-1) \\
-w_{E I} r_{I}(l)-\tilde{w}_{E I} r_{I}(l+1)-\tilde{w}_{E I} r_{I}(l-1)+i_{E}(l), \\
\mathcal{W}_{I}=w_{I E} r_{E}(l)+\tilde{w}_{I E} r_{E}(l+1)+\tilde{w}_{I E} r_{E}(l-1) \\
-w_{I I} r_{I}(l)-\tilde{w}_{I I} r_{I}(l+1)-\tilde{w}_{I I} r_{I}(l-1)+i_{I}(l),
\end{gathered}
$$

and where $l$ is the number (or, equivalently, the discrete spatial coordinate) of an excitatory-inhibitory node in the chain (Fig. 1). Variables $r_{E}(l)$ and $r_{I}(l)$ are the firing rates of the excitatory and inhibitory cells at node $l$, respectively, and $\tau_{E}$ is the characteristic time of excitatory cells (while time is normalized on the characteristic time of the inhibitory cells). Coefficients $w_{E E}, w_{E I}, w_{I E}$ and $w_{I I}$ describe the interactions of the excitatory and inhibitory cells within every node (as in [21]), and $\tilde{w}_{E E}, \tilde{w}_{E I}, \tilde{w}_{I E}, \tilde{w}_{I I}$ represent the strength of coupling between the nearest nodes. Inputs $i_{E}(l)$ and $i_{I}(l)$ of the excitatory and inhibitory cells are generated by the stimulation, while input ratio $i_{E}(l) / i_{I}(l)=\alpha$ is the same for all the nodes (although it could be modeled as an additional parameter). Below we focus on the linear regime of this system, where sigmoid function $g$ is approximated by $g(x) \approx x$. In other words, below we consider the following equations:

$$
\begin{aligned}
\tau_{E} \frac{d r_{E}(l)}{d t} & =-r_{E}(l)+\mathcal{W}_{E} \\
\frac{d r_{I}(l)}{d t} & =-r_{I}(l)+\mathcal{W}_{I}
\end{aligned}
$$


Figure 2. Spatial interference. (a) Stationary response $r_{E}(l)$ to a static point stimulus, where $i_{E}(0)=\alpha j$ and $i_{I}(0)=(1-\alpha) j$ with $\alpha=0.8, j=0.01$ and chain parameters $\tau_{E}=4, w_{E E}=2, w_{I E}=1.5, \tilde{w}_{E E}=\tilde{w}_{I E}=\tilde{w}_{E I}=1, \tilde{w}_{I I}=0.7$ (i.e., $\mathcal{K}=-1.2$ ), $\mathcal{T}=-0.8, \mathcal{M}=0.01$. Chain length was 200 nodes. The simulation time required to reach a stationary regime was 10000 with time step $d t=0.0001$. (b) Map of responses $r_{E}(l)$ across location $l$ for different distances $l_{1}$ and $l_{2}$ between two static point stimuli $i_{E}\left(l_{1}\right)=$ $i_{E}\left(l_{2}\right)=\alpha j$ and $i_{I}\left(l_{1}\right)=i_{I}\left(l_{1}\right)=(1-\alpha) j$, with all other currents set to zero. All other parameters are the same as in (a). Different rows of the map represent different simulations. The two yellow lines mark stimulus locations across simulations. Due to the neural wave interference, the middle point between the stimuli can be either facilitated (warm colors) or suppressed (cool colors), depending on the inter-stimulus distance and the spatial period of the wave of excitations. (c) Response $r_{E}(0)$ in the center of a "Gabor patch" stimulus as a function of spatial modulations period $n_{1}$, with all chain parameters as in (a) and with stimulus parameters in (10) set to $n_{0}=25$ and $j_{0}=0.0005$.
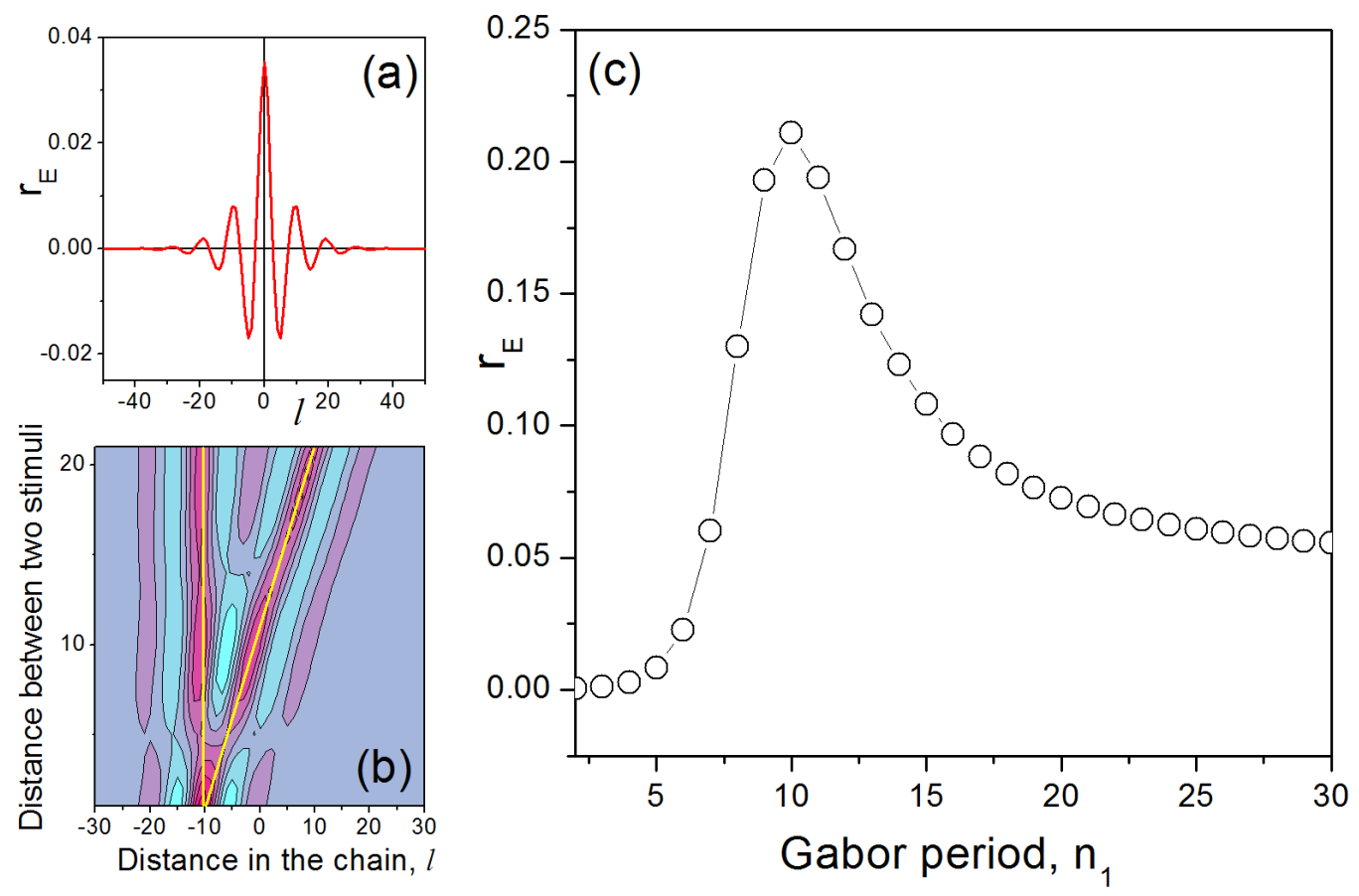

\section{Results and Discussion}

\subsection{Parameters responsible for network behavior}

Analyzing the stability of linearized equations (4), as well as static response of the network to point stimuli, we found [25] the parameters that lead to qualitatively different classes of network behavior, and which we summarize using the following coefficients:

$$
\mathcal{K}=4\left(\tilde{w}_{I I} \tilde{w}_{E E}-\tilde{w}_{E I} \tilde{w}_{I E}\right), \mathcal{R}=\tilde{w}_{E E}-\tau_{E} \tilde{w}_{I I},
$$




$$
\begin{gathered}
\mathcal{T}=\left(\tilde{w}_{E E}\left(w_{I I}+1\right)+\tilde{w}_{I I}\left(w_{E E}-1\right)-\tilde{w}_{E I} w_{I E}-\tilde{w}_{I E} w_{E I}\right) / \mathcal{K} . \\
\mathcal{Q}=w_{E E}-1-\tau_{E} w_{I I}-\tau_{E}+2\left|\tilde{w}_{E E}-\tau_{E} \tilde{w}_{I I}\right|<0, \\
\mathcal{M}=\left(w_{I I}+1\right)\left(1-w_{E E}\right)+w_{E I} w_{I E}+\mathcal{K} \mathcal{T}^{2}, \\
\tilde{k}= \pm \arccos (-\mathcal{T}), \quad \kappa= \pm \sqrt{-\mathcal{M} /\left[\mathcal{K}\left(1-\mathcal{T}^{2}\right)\right]} .
\end{gathered}
$$

In spite of the large number of parameters of the model, all stationary solutions (the spatial distribution of network activity) are controlled by two quantities, $\mathcal{T}$ and $\mathcal{M} / \mathcal{K}$, whereas the dynamical behavior of the network is controlled by $\mathcal{Q}$ and $\mathcal{R}$ as well. In particular, $\mathcal{T}$ determines whether the system has a purely decaying spatial response (for $|\mathcal{T}|>1$ ) or it shows decaying oscillations (for $|\mathcal{T}|<1$ ) with wave number $\tilde{k}$ (see, e.g., Fig. 2). Ratio $\mathcal{M} / \mathcal{K}$ defines the exponent of spatial decay (i.e., how fast the response decreases along the chain) away from the stimulus. Remarkably, when we choose different sets of parameters that correspond to the same magnitudes of $\mathcal{T}$ and $\mathcal{M} / \mathcal{K}$, but different magnitudes of $\mathcal{R}$, the network produces nearly the same responses for lasting stimuli, and very different responses for short-lived stimuli (Fig. 3).

\subsection{Spatial interference and intrinsic tuning}

First, we consider the steady-state response $r_{E}(l)$ to a spatially localized static stimulus ("point stimulus")

$$
i_{E}(l \neq 0)=i_{I}(l \neq 0)=0, i_{E}(0)=\alpha j, i_{I}(0)=(1-\alpha) j
$$

with total current $j$ applied to the zeroth node. Depending on parameters $\mathcal{T}$ and $\mathcal{M} / \mathcal{K}$, the response will have the form of either spatial damped oscillations (Fig. 2a) or purely exponential spatial decay (not shown). For the regime of damped oscillations, the number of significant oscillations (i.e., oscillations whose amplitudes are comparable to those at the locus of stimulation) is controlled by ratio $\tilde{k} / \kappa$ and it can be readily estimated using parameters $\mathcal{T}, \mathcal{M}$ and $\mathcal{K}$.

Applying two point stimuli, for which $i_{E}(l)=i_{I}(l)=0$ for any $l$ except $l_{1}$ and $l_{2}$, with

$$
i_{E}\left(l_{1}\right)=i_{E}\left(l_{2}\right)=\alpha j, i_{I}\left(l_{1}\right)=i_{I}\left(l_{2}\right)=(1-\alpha) j,
$$

we obtain an interference of two "static waves" generated by the two stimuli. Depending on distance $l_{1}-l_{2}$ between the stimuli, we obtain different interference responses $r_{E}(l)$, displayed in Fig. $2 \mathrm{~b}$ as a map in which the rows correspond to the simulations with different distances $l_{1}-l_{2}$.

Fig. $2 \mathrm{~b}$ reveals that the response at the middle position $l=\left(l_{1}+l_{2}\right) / 2$ between the point stimuli could be either facilitated (warm colors) or suppressed (cool colors). These results suggest a new interpretative framework for the experimental studies of visual sensitivity with different spatial arrangements of local stimuli, such as strings [9] or rings [10] of Gabor patches, which had revealed strong modulations of sensitivity between stimulus locations.

Next, consider the pattern of neuronal interference created by a spatially extended stimulus, such as the commonly used "Gabor patch"

$$
j(l)=j_{0} \cos \left(2 \pi l / n_{1}\right) \exp \left(-l^{2} / n_{0}^{2}\right)
$$


with spatial period $n_{1}$, Gaussian width $n_{0}$, and amplitude $j_{0}$. As we show in Fig. 2c, spatial period $n_{1}$ affects the response at the location of the strongest excitation $r_{E}(l=0)$. As spatial period $n_{1}$ increases, the response first increases, reaches a maximum, and then decreases. This effect can be interpreted as a constructive interference of neural waves. The maximal response $r_{E}\left(l=0, n_{1}\right)$ is reached when the periodicity of the stimulus matches the periodicity of the neural wave. In other words, the maximal response of the network is attained when a stimulus evokes oscillations whose period coincides with the period of the stimulus. (This period of oscillation may be called "intrinsic" or "natural" period of the network.)

Notably, the effect of inter-stimulus distance on the pattern of facilitation and suppression arises here from the nearest-neighbor interactions alone, requiring no long-range interactions between neurons.

\subsection{Spatiotemporal interference}

Next, we briefly consider the spatiotemporal interference that arises in the network when the stimuli appear at different locations and at different temporal instants. These conditions commonly occur in the studies that use short-lived and moving stimuli, where the latter may be equivalently described as sequential presentation of a short-lived stimulus at spatially adjacent locations.

The time course of chain response $r(l)$ after extinguishing the stimulus has the form of either a purely exponential temporal decay or a damped temporal oscillation. Here we focus on the response regime of temporal oscillation, which is similar to the response regime found in biological vision $[13,14]$.

We simulated a short-lived point excitation with $i_{E}(t, l)=i_{I}(t, l)=0$ for all $t$ and $l$ except $l=0, t<$ $t_{\text {stim }}$ when

$$
i_{E}\left(t<t_{\text {stim }}, l=0\right)=\alpha j, i_{I}\left(t<t_{\text {stim }}, l=0\right)=(1-\alpha) j .
$$

For $\mathcal{R}>0$, the slowest decaying response under $t>t_{\text {stim }}$ corresponds to the case, where all the nodes respond in phase (Fig. 3a,c) and the wave of neural excitation propagates with a very high velocity.

Interestingly, the maximum excitation at $r_{E}(t, l=0)$ is reached long after extinguishing the stimulus. (In Fig. 3, the stimulus was turned off at $t=1$ whereas $r_{E}$ reached its maximum at $t=20$.) Such response delays must be taken into account in the studies that use rapidly alternating or short-lived stimuli [26].

In case of $\mathcal{R}<0$, a long decay occurs for a fast spatial oscillating mode, resulting in out-of-phase oscillations in the neighboring neurons (Fig. 3b,d) and yielding a well-defined velocity of the neural wave. This makes the shape of the neural wave more complicated than in the case of $\mathcal{R}>0$, forming multiple regions of facilitation and suppression in the $l-t$ map (Fig. 3b) even for a single short pulse of stimulation. We therefore expect that different stimuli (not shown) will generate non-trivial interference patterns. For example, consider network response to a dynamic stimulus, a drifting Gabor patch:

$$
j(l)=j_{0} \cos \left(2 \pi\left(l-v_{g} t / n_{1}\right)\right) \exp \left(-l^{2} / n_{0}^{2}\right)
$$

The well-defined velocity of the wave for $\mathcal{R}<0$ suggests that the interference pattern will generate clear maxima of $r_{E}(l)$ when the stimulus moves with the same velocity as the intrinsic ("natural") velocity of the neural wave. This result agrees with the numerical stimulations illustrated in Fig. $3 \mathrm{~g}$, and it suggests a simple neural mechanism for tuning the sensory system to stimulus velocity, as a special case 
of spatiotemporal neural-wave interference, without the need to posit a specialized neural circuit for sensing velocity. Notably, and in spite of the very different spatiotemporal responses of the system to short-lived stimuli (Fig. 3a,b), responses to static stimuli for $\mathcal{R}>0$ and $\mathcal{R}<0$ are similar to one another (Fig. 3e,f) because the steady-state response of the network is fully determined by terms $\mathcal{K}, \mathcal{T}$ and $\mathcal{M}$.

Figure 3. Spatiotemporal interference. (a-b) Map of spatiotemporal response $r_{E}(t, l)$ to short-lived point stimulus, where $i_{E}(t, l)=i_{I}(t, l)=0$ for all $l \neq 0 ; i_{E}\left(t<t_{\text {stim }}, 0\right)=$ $\alpha j, i_{I}\left(t<t_{\text {stim }}, 0\right)=(1-\alpha) j$ and $i_{E}\left(t>t_{\text {stim }}, 0\right)=0, i_{I}\left(t>t_{\text {stim }}, 0\right)=0$. The abscissa is spatial coordinate $l$ and the ordinate is time $t$ after stimulus onset $r_{E}(0, i)=r_{I}(0, i)=0$. The red, cyan, and blue regions represent, respectively, high positive, near zero, and large negative values of $r_{E}$. Chain parameters were $w_{E E}=2, w_{I E}=1.5, \mathcal{M}=0.01, \mathcal{Q}=-0.01$, $\mathcal{T}=-0.8, \mathcal{K}=-0.1, \alpha=0.8$ for both (a) and (b), where $\mathcal{R}=1$ (a), $\tilde{w}_{E E}=1.3$ (a), $\tilde{w}_{I E}=1.7$ (a) and $\mathcal{R}=-1$ (b) $\tilde{w}_{E E}=1.5$ (b), $\tilde{w}_{I E}=1.6$ (b). Chain length was 200 nodes. Simulation time was 40 , and the time step was $d t=10^{-6}$. Stimulus duration was 1 and stimulus intensity was $j=4 \times 10^{-4}$. (c-d) Results of the same simulations as in (a) and (b), respectively, now displaying functions $r_{E}(t, 0), r_{E}(t, 1) \ldots r_{E}(t, 5)$. The nodes oscillate in phase when $\mathcal{R}>0$ and out of phase when $\mathcal{R}<0$. (e-f) Steady-state responses for a static point stimulus $i_{E}(t, l)=i_{I}(t, l)=0$ and $i_{E}(t, 0)=\alpha j, i_{I}(t, 0)=(1-\alpha) j$ for the same chain parameters as in (a) and (b), respectively. (Stimulus intensity was $j=4 \times 10^{-4}$.) In spite of the different chain responses for the short-lived and lasting stimuli (due to the different signs of $\mathcal{R}$ ), the steady-state responses are nearly identical, except for a slightly different amplitude $r_{E}(l=0)$, since the steady-state response is controlled only by $\mathcal{T}, \mathcal{M}, \mathcal{K}$. (g) Maximal response $\max _{t}\left[r_{E}(t, l=0)\right]$ over the entire observation period on the central node for a drifting Gabor patch (11) with parameters $n_{1}=2, n_{0}=20, j_{0}=0.0005$. The response is stronger than for static stimuli $\left(v_{g}=0\right)$.
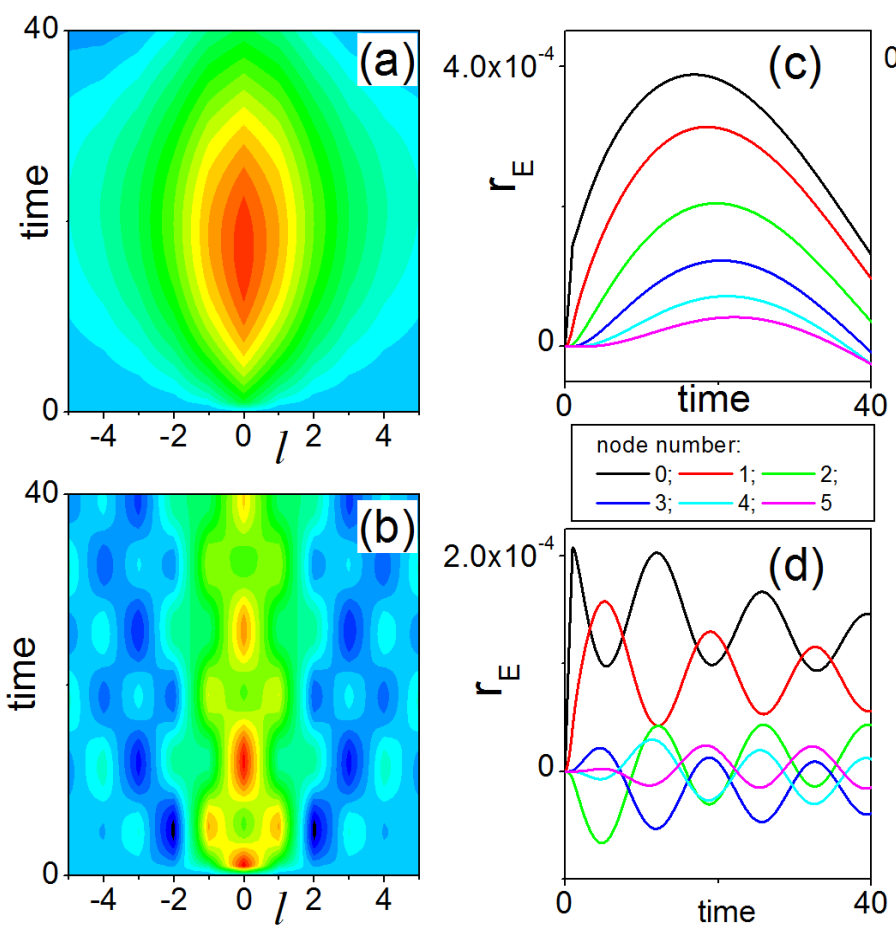
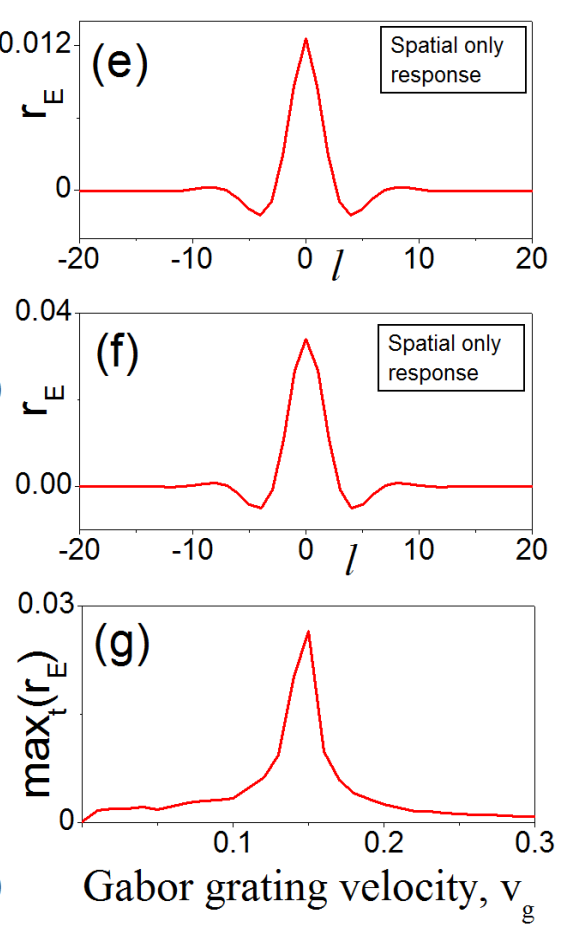


\section{Conclusions}

We analyzed interference of the neural waves propagating through an inhibitory-excitatory neural chain with nearest-neighbor coupling. We derived the combinations of parameters responsible for the shape of neural waves generated by static and short-lived stimuli. We found that the interference patterns generated in this network have three properties commonly observed in biological vision: (1) selectivity for to spatial and temporal frequencies of stimulus intensity modulation, (2) selectivity for stimulus velocity, and (3) "lateral interactions" between spatially separate stimuli. It is possible that neural wave interference is responsible for some of the basic visual phenomena that had been attributed to distinct and specialized neural mechanisms.

\section{Acknowledgements}

SS acknowledges support from the Leverhulme Trust.

\section{Author Contributions}

SS and SG conceived the idea, designed the study and wrote the paper. SS performed computer simulations.

\section{Conflicts of Interest}

The authors declare no conflict of interest.

\section{References}

1. Landau, L.D.; Lifshitz, E.M. Fluid mechanics; Butterworth-Heinemann: Oxford, UK, 2010.

2. Landau, L.D.; Lifshitz, E.M. The classical theory of fields; Butterworth-Heinemann: Oxford, UK, 2010.

3. Everest, F.A.; Pohlmann, K.C. Master handbook of acoustics; McGraw-Hill/TAB Electronics: USA, 2009.

4. Rossing, T.; Chiaverina, C.J. Light science: physics and the visual arts; Springer: New York, USA, 1999.

5. Landau, L.D.; Lifshitz, E.M. Quantum mechanics, non-relativistic theory; Butterworth-Heinemann: Oxford, UK, 2010.

6. Ficek, Z.; Swain, S. Quantum interference and coherence: theory and experiments; Springer New York, USA, 2010.

7. Ermentrout, B. Neural networks as spatiotemporal pattern-forming systems. Rep. Prog. Phys. 1998, 61, 353-430.

8. Bressloff, P.C. Spatiotemporal dynamics of continuum neural fields. J. Phys. A: Math. Theor. 2012, 45, 033001.

9. Polat, U.; Sagi, D. Lateral interactions between spatial channels: suppression and facilitation revealed by lateral masking experiments. Vision Res. 1993, 33, 993-997. 
10. Kovacs, I.; Julesz, B. Perceptual sensitivity maps within globally defined visual shapes. Nature 1994, 370, 644-646.

11. Adini, Y.; Sagi, D.; Tsodyks, M. Excitatory-inhibitory network in the visual cortex: psychophysical evidence. Proc. Natl. Acad. Sci. U.S.A. 1997, 94, 10426-10431.

12. Gilbert, C.D.; Das, A.; Ito, M.; Kapadia, M.; Westheimer, G. Spatial integration and cortical dynamics. Proc. Natl. Acad. Sci. U.S.A. 1996, 93, 615-622.

13. Manahilov, V. Spatiotemporal visual response to suprathreshold stimuli. Vision Res. 1995, 35, 227-237.

14. Manahilov, V. Triphasic temporal impulse responses and Mach bands in time. Vision Res. 1998, 38, 447-458.

15. Jones, A.J.; Palmer, L. An evaluation of the two-dimensional Gabor filter model of simple receptive fields in cat striate cortex. J. Neurophys. 1987, 58, 1233-1258.

16. Marr, D. Vision: A computational investigation into the human representation and processing of visual information; W.H. Freeman Ed.; MIT Press: USA, 2010.

17. Poggio, T.; Girosi, F. Regularization algorithms for learning that are equivalent to multilayer networks. Science 1990, 247, 978-982.

18. Stevens, C.F. What form should a cortical theory take?; In Large-scale neuronal theories of the brain; Koch, C.; Davis, J.L., Eds.; The MIT Press: USA, 1994; pp. 239-255.

19. Wilson, H.R. Spikes, decisions, and actions: The dynamical foundations of neuroscience; Oxford University Press: Oxford, UK, 1999.

20. Wilson, H.R.; Cowan, J.D. A Mathematical theory of the functional dynamics of cortical and thalamic nervous tissue. Kybernetik 1973, 13, 55-80.

21. Ozeki, H.; Finn, I.M.; Schaffer, E.S.; Miller, K.D.; Ferster, D. Inhibitory Stabilization of the Cortical Network Underlies Visual Surround Suppression. Neuron 2009, 62, 578-592.

22. Tsodyks, M.V.; Skaggs, W.E.; Sejnowski, T.J.; McNaughton, B.L. Paradoxical Effects of External Modulation of Inhibitory Interneurons. J. Neurosci. 1997, 17, 4382-4388.

23. Bragin, A.; Jando, G. ; Nadasdy, Z.; Hetke, J.; Wise, K.; Buzsaki, G. Gamma (40-100 Hz) oscillation in the hippocampus of the behaving rat. J. Neurosci. 1995, 15, 47-60.

24. Jadi, M.P.; Sejnowski, T.J. Regulating cortical oscillations in an inhibition-stabilized network. Proc. IEEE, 2014, 102, 830-842.

25. Saveliev, S.; Gepshtein S., Spatial and Temporal Interference of Neural Waves in Inhibition-Stabilized Networks. Unpublished.

26. Tadin, D.; Lappin, J.S.; Gilroy, L.A.; Blake, R. Perceptual consequences of center-surround antagonism in visual motion processing. Nature 2003, 424, 312-315.

(c) by the authors; licensee MDPI, Basel, Switzerland. This article is an open access article distributed under the terms and conditions of the Creative Commons Attribution license (http://creativecommons.org/licenses/by/3.0/). 